\title{
Mothers' attitudes to preterm infants
}

\author{
R. T. BIDDER,* E. A. CROWE, $\uparrow$ and O. P. GRAY \\ From the Department of Child Health, Welsh National School of Medicine, Heath Park, Cardiff, Wales
}

\begin{abstract}
Bidder, R. T., Crowe, E. A., and Gray, O. P. (1974). Archives of Disease in Childhood, 49, 766. Mothers' attitudes to preterm infants. Mothers' attitudes to their preterm and term children were examined and compared using the SemanticDifferential and an interview related to the experiences of mothers during pregnancy and after birth. It was postulated that mothers would show more concern for their preterm infant than their term infant, but this hypothesis was not supported by the results. The attitudes of mothers were analysed and the only statistical significance was in the concept 'strong-weak', which indicated that mothers see their preterm child as being weaker than their term child. The interview material revealed interesting additional findings that there were two specific periods of concern, and also elaborated the 'strong-weak' concept.
\end{abstract}

There are many adverse pathological processes affecting the low birthweight infant which may be responsible for its poorer outlook when compared with the term infant. There is an increased incidence of birth asphyxia, hypoglycaemia, hypocalcaemia, convulsions, birth trauma, and hypothermia (Drillien, 1964), though fortunately the incidence of many of these has been reduced in recent years and the prognosis for the low birthweight infant is steadily improving. One possible adverse process affecting the child's development, which has recently been the subject of investigation, is the altered mother/child relationship in the early hours and days. Klaus et al. (1972) have suggested that there may be a special attachment period immediately after birth. This period is important for the mother because what happens during this time may affect her later behaviour towards the infant. Klaus et al. suggested that this period deserves a special name-the 'maternal sensitive period'. Increased maternal attentiveness during this period encourages the infant's exploratory behaviour and the development of cognitive behaviour. Klaus et al. showed that early and extended contact by the mother with her infant at this time may affect her interaction with the infant for at least 30 days and possibly a year.

In contrast to the mother of the term infant, the

Received 27 March 1974.

Present addresses: *Preswylfa Child and Family Centre, Clive Road, Canton, Cardiff.

tSt. Cadoc's Hospital, Caerleon, Gwent. mother of a preterm infant has problems not only in making these early bonds of attachment, but also those arising from the emotional crisis surrounding the preterm delivery. In the preterm infant the mother has much less opportunity to handle her baby and the period of separation is greatly extended. It may be impossible for several weeks for her to have more than a hand-to-hand contact of brief duration through an incubator port. Furthermore, the length of time that she spends with her infant during these early weeks is much reduced. Sometimes the mother may not be welcomed at frequent intervals during the day and she may be required to adopt procedures such as gowning and masking which tend to discourage her visiting. In addition to the incubator barrier, the infant itself may discourage the mother. He may appear physically unattractive, fragile, wasted, and be inattentive towards the mother. He may be ill and thereby cause considerable maternal anxiety. The preterm birth is associated with a hospital atmosphere more characteristic of an emergency than a normal birth, and the mother is aware of the risks to her child. Frequently she is discharged home long before the infant.

Kaplan and Mason (1960) believe that the mother has four psychological tasks to perform when birth is premature. She has to prepare herself for the possible loss of the child whose life is in jeopardy; this is the so-called anticipatory grief. Secondly, she has to come to terms with her failure to deliver a normal baby; this can result in depression. Later, 
when it appears that the baby is likely to survive, she has to resume the normal mother/infant relationship. Finally, she has to acknowledge the special needs of the preterm infant as a temporary state, later to be treated as normal.

Smith et al. (1969) used an interview and rating scale technique to explore the preterm infant mother's mood, her acceptance of the pregnancy, the mothering role, and the concern for the infant's welfare. They found no significant differences between the mothers of the preterm infants and the mothers of term infants in the early post-partum period. However, others (Mussen, Conger, and Kagan, 1969) have stated that parents continue to regard an infant born preterm as needing extra care and attention. A continuing disturbed attitude towards a preterm infant has been suggested by the fact that battered babies are found more commonly among those born prematurely (Klein and Stern, 1971).

We have investigated the hypothesis that a mother of a preterm infant will have more concern for this infant than for her term infant and that this concern extends beyond the period of immediate risk up to 2 or 3 years. We have also investigated the hypothesis that the mother perceives her own child to be different from her notion of an ideal child. This was done using Hurlock's (1964) concept of the ideal child.

\section{Material and methods}

The 20 mothers selected for this study were those who had at least 2 children, one born preterm-less than 37 weeks' gestation - and the other born at term, who served as the control infant. 11 of the control children were older than the index cases and 9 were younger. The mean age at interview of the preterm infants was 2.8 years and that of the term infants 3.5 years. There were no perinatal complications among the control children. Every sixth preterm infant admitted to the Special Care Unit was selected for study. Children with physical abnormalities were excluded. Permission for inclusion in this study was sought from the mothers who were later visited at their home for the investigation. There was no refusal. The concept of an ideal child was not defined for the mothers. They interpreted this phrase themselves. The degree of maternal concern was measured by the Semantic-Differential (Osgood, Suci, and Tannenbaum, 1957). This technique is a combination of association and scaling procedures, designed to give an objective measure of the connotative meaning of a concept. Selective concepts are rated by the mother on a series of 7-point scales, each defined by adjectives which are opposite in meaning. The mothers used this concept for the children, and half completed the scale in the order of term, preterm, and ideal, and the other half used the order of preterm, term, and ideal child. The 20 scales are shown in Table I. They were designed to answer the questions-do the mothers hold different attitudes to their preterm and term infant and, if so, is the preterm infant seen to be in need of more attention and care, and does the mother see either of her children as different from the ideal child?

All the mothers were interviewed at home and given the Semantic-Differential scales to complete for both the index and control child. After this they were asked questions relating to their experience of pregnancy, the

TABLE I

Means of the ratings on each scale for the 3 groups of preterm $(P T)$, term $(T)$, and ideal child (ID)

\begin{tabular}{|c|c|c|c|c|c|c|}
\hline \multirow[t]{2}{*}{ Scale } & \multicolumn{3}{|c|}{ Means } & \multicolumn{3}{|c|}{$\begin{array}{l}\text { Statistically significant differences between } \\
\text { groups (Sign Test) }\end{array}$} \\
\hline & PT & $\mathbf{T}$ & ID & $\mathrm{PT}-\mathrm{T}$ & PT-ID & $T-I D$ \\
\hline $\begin{array}{l}\text { 1. Delicate-Tough } \\
\text { 2. Eating well-Choosy } \\
\text { 3. Rough-Gentle } \\
\text { 4. Confident-Timid } \\
\text { 5. Anxious-Relaxed } \\
\text { 6. Noisy-Quiet } \\
\text { 7. Happy-Unhappy } \\
\text { 8. Insecure-Secure } \\
\text { 9. Dependent-Independent } \\
\text { 10. Bad-Good } \\
\text { 11. Calm-Excitable } \\
\text { 12. Strong-Weak } \\
\text { 13. Submissive-Aggressive } \\
\text { 14. Active-Passive } \\
\text { 15. Isolated-Sociable } \\
\text { 16. Generous-Selfish } \\
\text { 17. Dissatisfied-Satisfied } \\
\text { 18. Warm-Cold } \\
\text { 19. Hard-Soft } \\
\text { 20. Worries me-Does not }\end{array}$ & $\begin{array}{l}4 \cdot 4 \\
3 \cdot 0 \\
3 \cdot 9 \\
3 \cdot 4 \\
3 \cdot 9 \\
2 \cdot 6 \\
2 \cdot 3 \\
5 \cdot 5 \\
3 \cdot 1 \\
5 \cdot 1 \\
5 \cdot 2 \\
3 \cdot 0 \\
4 \cdot 7 \\
1 \cdot 8 \\
5 \cdot 8 \\
2 \cdot 3 \\
5 \cdot 0 \\
2 \cdot 1 \\
4 \cdot 4 \\
4 \cdot 7\end{array}$ & $\begin{array}{l}4 \cdot 8 \\
3 \cdot 2 \\
4 \cdot 2 \\
4 \cdot 0 \\
4 \cdot 2 \\
2 \cdot 4 \\
2 \cdot 5 \\
5 \cdot 3 \\
2 \cdot 8 \\
5 \cdot 1 \\
4 \cdot 8 \\
2 \cdot 3 \\
4 \cdot 2 \\
2 \cdot 4 \\
5 \cdot 5 \\
2 \cdot 9 \\
4 \cdot 5 \\
2 \cdot 3 \\
4 \cdot 6 \\
5 \cdot 2\end{array}$ & $\begin{array}{l}5 \cdot 4 \\
1 \cdot 8 \\
3 \cdot 8 \\
2 \cdot 6 \\
5 \cdot 4 \\
4 \cdot 0 \\
1 \cdot 7 \\
5 \cdot 3 \\
2 \cdot 6 \\
5 \cdot 6 \\
3 \cdot 8 \\
2 \cdot 5 \\
3 \cdot 9 \\
2 \cdot 1 \\
6 \cdot 1 \\
2 \cdot 1 \\
5 \cdot 8 \\
1 \cdot 8 \\
4 \cdot 2 \\
5 \cdot 6\end{array}$ & $<0.05$ & $\begin{array}{l}<0.001 \\
<0.005 \\
<0.05\end{array}$ & $\begin{array}{l}<0.01 \\
<0.005 \\
<0.005\end{array}$ \\
\hline
\end{tabular}


infant's birth, period in hospital, time at home, and the infant's early development and progress. The questions were asked for both children separately. All the 20 interviews were tape-recorded. The information obtained from each interview was evaluated in terms of the categories (shown in Table II) by four independent raters, two psychologists (one male and one female) and two social workers (one male and one female) who did not know whether the child was a control or index case. Independent agreement by at least three out of the four raters was necessary in order for an item to be placed into a particular category. That there was very good agreement among the raters is shown in Table II. When there was any disagreement the sum of the items was less than 20 .

\section{Results}

The differences between the ratings on each of the scales were analysed for significance using the sign test (Siegel, 1956) (Table I). Mothers saw their preterm children as weaker than their term children (scale 12 : Strong-Weak; $\mathbf{P}<0 \cdot 05$ ). There are significant differences between the preterm and term infants and the ideal child. Mothers saw the ideal child to be more relaxed, quieter, calmer, and more satisfied than either their preterm or term child.

The preterm group was divided into two groups according to whether they were born before or after the term child. No significant differences were found on any of the scales, nor were there any significant differences attributable to the sex of the preterm child.

The analysis of the interview material (Table II) was conducted using Fisher's exact probability test (Siegel, 1956). There were no differences between the mother's reaction to the pregnancy of either the preterm or the term infant. The mother reported no difference in the physical experience of pregnancy or birth. The only differences noticed were that immediately after birth the mothers were considerably more anxious about their preterm infant than about their term infant $(P<0.005)$. The mothers were more anxious also when looking after their preterm infant at home than when looking after the term infant. They could not remember any difference in the amount of support received from either professional or family sources when looking after both infants at home.

The mothers felt there were no differences between the rates of development of the two groups of children. They were no more worried about their preterm infants than about their term infants at the time of the study.

Further analysis was carried out to see if early experience influenced the mother's rating of her preterm child on the Semantic-Differential. Each interview category was compared with each of the

TABLE II

Analysis of interview material

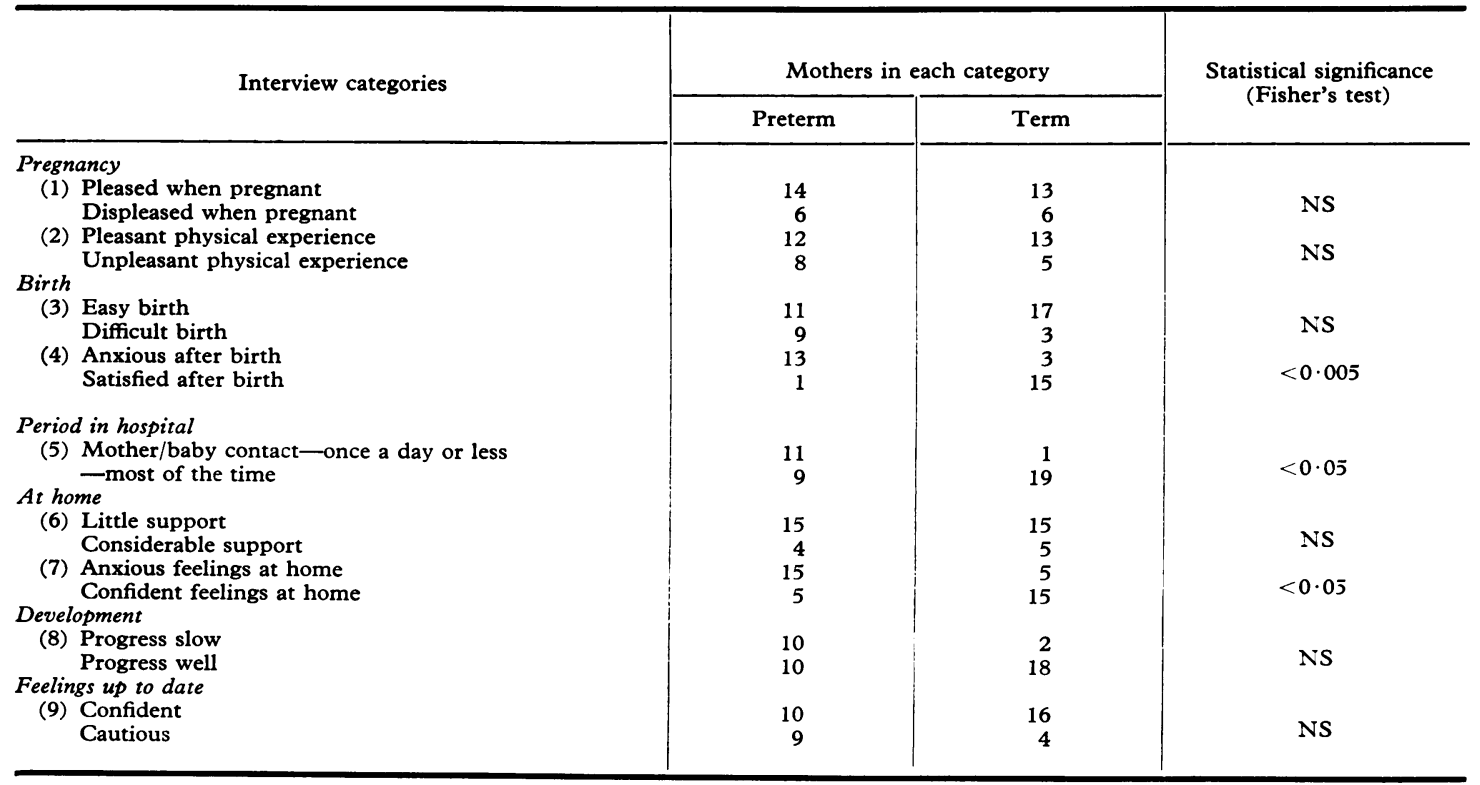


scales of the Semantic-Differential. For instance, when the mother reported feeling anxious at the birth of her preterm baby, but not anxious about the birth of her term baby, her ratings of the preterm baby on the Semantic-Differential were compared with her ratings of the term infant. The sign test was used to evaluate the significance of the effect of early experience on mother's ratings of her children. The concept strong-weak reveals significant differences and some tendencies; the mothers of preterm infants who were anxious about their infant at birth now see the child as weak $(P<0 \cdot 005)$. When the mothers were anxious in handling their baby at home they now view their infant as weak $(\mathbf{P}$ $<0 \cdot 05)$. Mothers who were displeased when they learned that they were pregnant see their preterm baby as weaker than their term $(P<0.05)$, and mothers who felt that they had had little support tend to view their preterm child as weak.

\section{Discussion}

This study was begun with the belief that mothers would be more concerned for their preterm than their term children. Several differences were expected, but the remarkable finding was that by the third year statistical significance was found only in relation to the concept 'strong-weak'. Mothers see their preterm child as being weaker than their term child. There are several possible reasons for this phenomenon. The sample, though small, was sufficiently large to show differences should they be present. It is unlikely that the results are due to deficiency in technique, because the SemanticDifferential is specifically designed to detect attitudinal differences. The most likely explanation is that the considerable support received from the health visitor allayed the mother's natural anxiety about the preterm infant.* Caplan (1960) and Warrick (1971) emphasized the importance of expert help in producing a healthy outcome after the birth of a preterm infant. The interview material shows that there are two periods when mothers are especially anxious about the preterm infant. One is immediately after birth, and the other is upon the infant's return home, showing that they are aware of the implications of prematurity, contrary to the findings of Smith et al. (1969).

The mothers' interviews reveal that their anxieties centre around practical problems such as the difficulty of handling a small, delicate child; its susceptibility to infection and illness; and feeding

\footnotetext{
*It is the practice in the City of Cardiff for the health visitor seconded to the care of the mothers of preterm babies to make frequent visits immediately after the infant is discharged from hospital.
}

problems. Even though the mothers did receive specialist help, 14 expressed the need for even more support and did not remember any difference in the amount and type of support received for preterm and term infant. It is possible that the period spent at home looking after the preterm infant generated so much anxiety that the amount of specialist help received both at hospital and at home was quickly forgotten.

Our additional hypothesis that the mother's perception of the ideal child will be different from that of her own term or preterm child was supported by the present findings. The traditional adult concept of the ideal child is one who respects his parents, obeys, pleases adults, and shares, and co-operates in the duties of the home (Hurlock, 1964). The mothers in the present study saw their own children as less confident, less relaxed, less quiet, less calm, less generous, and less satisfied than the ideal child.

This study does not support the view that the birth of a preterm infant leads to a permanently altered mother/child relationship, but it does indicate two specific periods when the mother is most anxious: immediately after birth and when the baby goes home. As a result, even in the third year the mother sees the preterm infant as weaker than the term infant. This implies that effort should be concentrated on minimizing the anxiety experienced by the mothers during these periods.

We thank Dr. D. G. Melville Thomas, Consultant Child Psychiatrist, for his support; Mrs. T. Keogh, Health Visitor; Mrs. A. Turner and Mr. R. Davies, Social Workers; Mrs. J. Sheehan and Mr. P. Holden, Clinical Psychologists, for their help; and Mrs. S. Josty for the preparation of the manuscript; and above all the mothers who participated in this study.

\section{REFERENCES}

Caplan, G. (1960). Patterns of parental response to the crisis of premature birth. Psychiatry, 23, 365.

Drillien, C. M. (1964). The Growth and Development of the Prematurely Born Infant. Livingstone, Edinburgh.

Hurlock, E. B. (1964). Child Development, 4th ed. McGraw-Hill, New York.

Kaplan, D. M., and Mason, G. A. (1960). Maternal reaction to premature birth viewed as an acute emotional disorder. American fournal of Orthopsychiatry, 30, 539.

Klaus, M. H., Jerauld, R., Kreger, N. C., McAlpine, W., Steffa, M., and Kennell, J. H. (1972). Maternal attachment: importance of the first post-partum days. New England fournal of Medicine, 286, 460 .

Klein, M., and Stern, L. (1971). Low birth-weight and the battered-child syndrome. American fournal of Diseases of Children, 122, 15.

Mussen, P. H., Conger, J. J., and Kagan, J. (1969). Child Development and Personality. Harper International, New York.

Osgood, G. E., Suci, G. T., and Tannenbaum, P. (1957). The Measurement of Meaning. University of Illinois Press, Urbana, Illinois. 
Siegel, S. (1956). Non-Parametric Statistics for the Behavioral Sciences. McGraw-Hill, New York.

Smith, N., Schwartz, J. R., Mandell, W., Silberstein, R. M., Dalack, J. D., and Sacks, S. (1969). Mothers' psychological reactions to premature and full-size newborns. Archives of General Psychiatry, 21, 171.
Warrick, L. H. (1971). Family-centred care in the premature nursery. American fournal of Nursing, 71, 2134.

Correspondence to Professor O. P. Gray, Department of Child Health, Welsh National School of Medicine, Heath Park, Cardiff CF4 4XW. 